\title{
A genetic algorithm for solving supply chain network design model
}

\begin{abstract}
Network design is by nature costly and optimization models play significant role in reducing the unnecessary cost components of a distribution network. This study proposes a genetic algorithm to solve a distribution network design model. The structure of the chromosome in the proposed algorithm is defined in a novel way that in addition to producing feasible solutions, it also reduces the computational complexity of the algorithm. Computational results are presented to show the algorithm performance.
\end{abstract}

Keyword: Distribution network design; Genetic algorithm; Meta-heuristic algorithm; Supply chain management 